\title{
Publisher Correction: Stochastic comparisons of order statistics from scaled and interdependent random variables
}

\author{
Chen $\mathrm{Li}^{1} \cdot$ Rui Fang ${ }^{2} \cdot$ Xiaohu $\mathrm{Li}^{3}$
}

Published online: 8 March 2018

(C) Springer-Verlag GmbH Germany, part of Springer Nature 2018

\section{Correction to: Metrika (2016) 79:553-578 https://doi.org/10.1007/s00184-015-0567-3}

In the original publication of the article, the article title was incorrectly published as "Stochastic somparisons of order statistics from scaled and interdependent random variables". However, the correct title should read "Stochastic comparisons of order statistics from scaled and interdependent random variables".

The original article can be found online at https://doi.org/10.1007/s00184-015-0567-3.

\section{Xiaohu Li}

mathxhli@hotmail.com; xiaohu.li@stevens.edu

1 School of Mathematical Sciences, Xiamen University, Xiamen 361005, Fujian, China

2 Department of Mathematics, Shantou University, Shantou 515063, Guangdong, China

3 Department of Mathematical Sciences, Stevens Institute of Technology, Hoboken, NJ 07030, USA 